\title{
Computation of Thermal Expansion Coefficient of Graphene with Gaussian Approximation Potentials
}

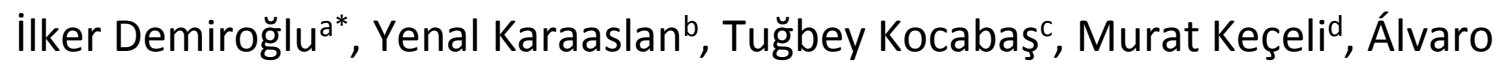
Vázquez-Mayagoitiad, and Cem Sevik ${ }^{\mathrm{b}, \mathrm{e}^{*}}$

a Department of Advanced Technologies, Eskisehir Technical University, Eskisehir, TR 26555, Turkey.

${ }^{\mathrm{b}}$ Department of Mechanical Engineering, Eskişehir Technical University, Eskisehir, TR 26555, Turkey.

c Department of Materials Science and Engineering, Institute of Graduate Programs, Eskişehir Technical University, Eskisehir, TR 26555, Turkey.

${ }^{d}$ Computational Science Division, Argonne National Laboratory, Lemont, IL 60439, USA.

e Department of Physics, University of Antwerp, Groenenborgerlaan 171, 2020 Antwerp, Belgium

*E-mails: ilkerdemiroglu@eskisehir.edu.tr, csevik@eskisehir.edu.tr 

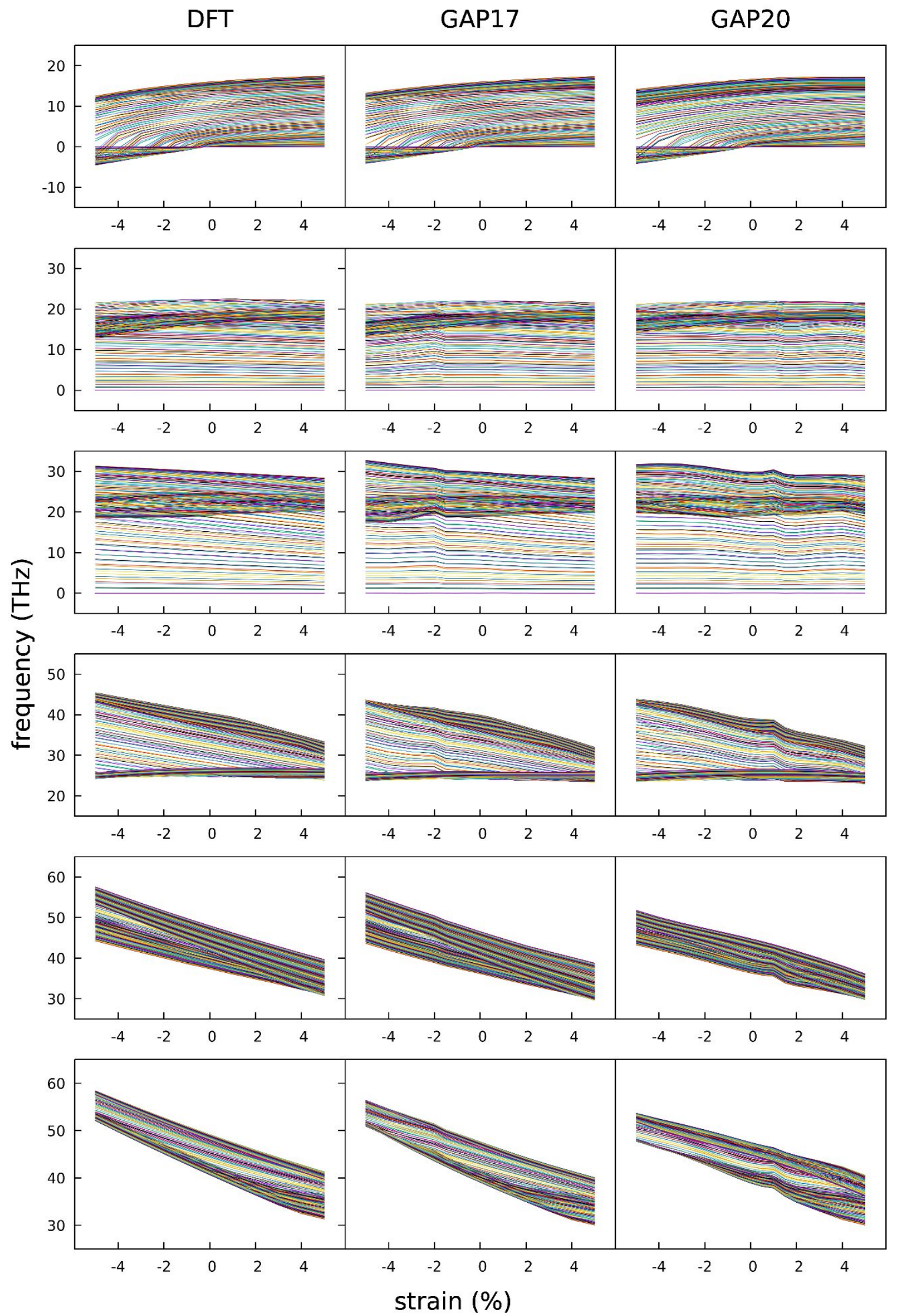

Figure S1. All the calculated phonon frequencies and how they change w.r.t applied strain up to $5 \%$ strain. 

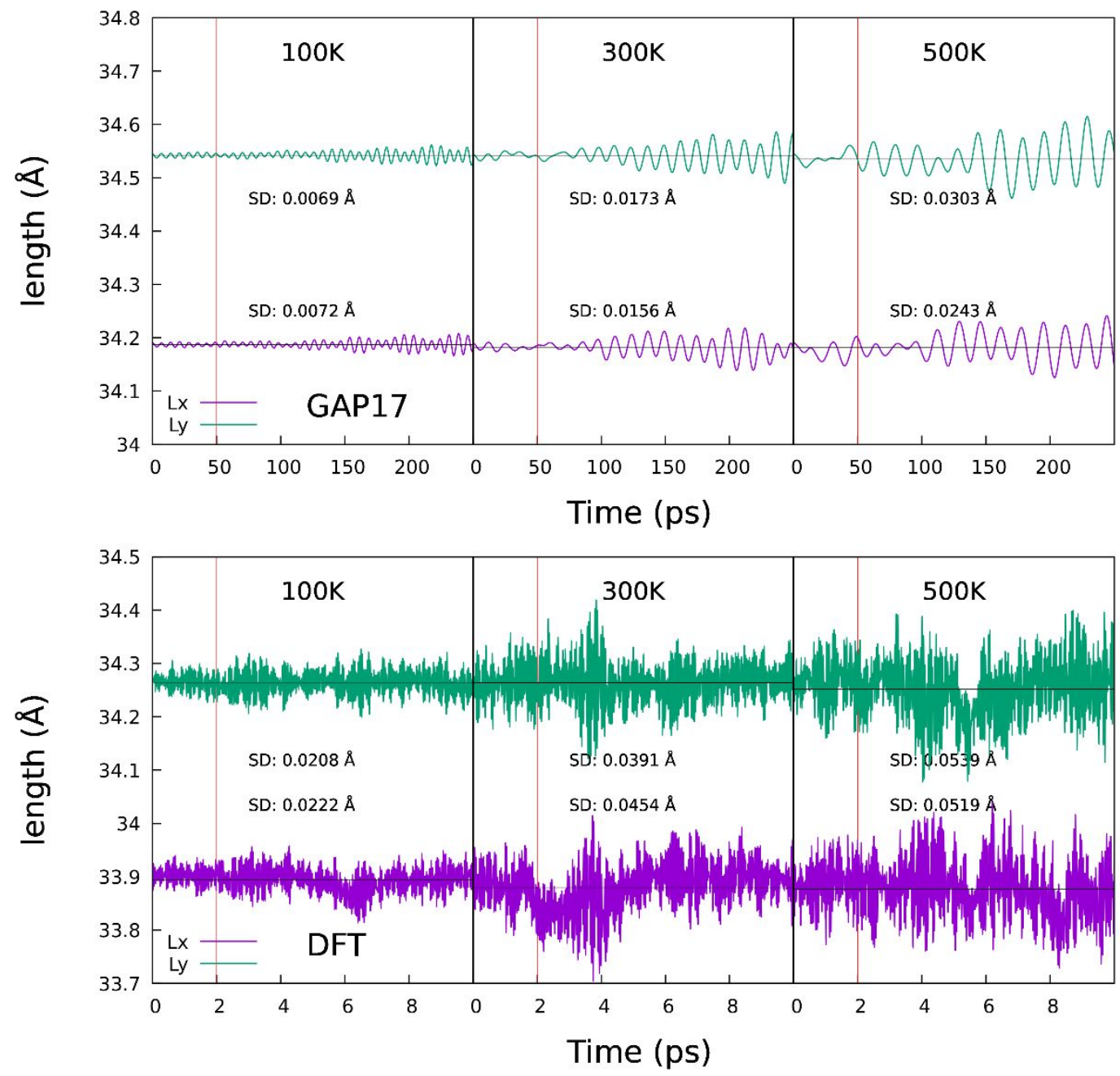

Figure S2. The variation in length of $\mathrm{x}$ and y vectors (Lx and Ly) during GAP17 (top) and DFT (bottom) MD simulations for 448 atom unit cell size. Standard deviation (SD) values are indicated on the graph and the mean values are given as straight lines. Equilibration phases of MD simulations are indicated by the red vertical lines (2 ps with DFT and 50 ps with GAP17). 